\title{
Middle lobe syndrome in the left lower lobe in chronic obstructive pulmonary disease
}

\author{
*Allen J Blaivas, Walter Strauss \\ Assistant Professor of Medicine, Department of Pulmonary, Critical Care, and Sleep Medicine, VA New Jersey Health Care System, \\ East Orange, New Jersey, USA
}

Received 12th September 2008; revised version received 22nd December 2008; accepted 31st January 2009; online 10th June 2009

\author{
Summary \\ Middle lobe syndrome is a term that refers to a recurrent collapse of a lung segment, typically the right middle lobe. We present here a \\ case of middle lobe syndrome which occurred in an unusual lung segment and which was related to an unusual cause. \\ (C) 2009 General Practice Airways Group. All rights reserved. \\ AJ Blaivas an W Strauss. Prim Care Resp J 2009; 18(4): 331-333 \\ doi:10.4104/pcrj.2009.00018
}

Keywords exacerbation, COPD, pneumonia, infection

\section{Introduction}

Middle lobe syndrome (MLS) refers to chronic or recurrent radiographic evidence of collapse of the right middle lobe (RML). Although termed the "middle lobe syndrome", it does occur less commonly in other areas of the lung, particularly in children with asthma. ${ }^{1}$ We describe a case of MLS in the left lower lobe of a patient with moderate chronic obstructive pulmonary disease (COPD).

\section{Case presentation}

A 49 year-old man with a past medical history significant for moderate COPD and frequent pulmonary exacerbations was referred to the pulmonary clinic. He had experienced frequent "pneumonias" associated with left lower chest pain and cough productive of purulent sputum intermittently over a number of years. He denied fevers during these events, but stated that he tended to have concurrent drenching nighttime sweats. Despite this he continued to smoke. He noted exertional dyspnoea, with a NYHA functional classification of class II. He denied any significant history of asthma, tuberculosis, or recent occupational exposure, though he did admit to a brief stint of employment in a chemical manufacturing plant many years ago. He also suspected that he had had asbestos exposure in the past. His physical examination was only significant for diffuse mild wheezing. Spirometry revealed the following post-bronchodilator lung function; FEV 12.72 liters (59\% predicted), FVC 4.42 liters (75\% predicted), and an $\mathrm{FEV}_{1} / \mathrm{FVC}$ ratio of 0.62 . He was started on treatment for his COPD with a combination inhaled corticosteroid and long-acting beta-agonist in addition to the previously prescribed ipratropium.

A computed tomography (CT) scan (Figure 1) revealed atelectasis of the superior segment of the left lower lobe (LLL) that seemed to be slightly increased in size compared to a previous CT scan done in 1994. Of note, there was no radiographic evidence of bronchiectasis. Bronchoscopy was performed to rule out an endobronchial lesion; the airways were all normal, with the exception of the superior segment of the left lower lobe where the airway was somewhat oedematous with whitish thick secretions partially obscuring it. The secretions were suctioned and bronchoalveolar lavage (BAL) was performed. The subsequent bacterial and AFB cultures and cytologic specimens were negative. The patient tolerated the bronchoscopy without incident and postprocedure chest $x$-ray showed persistence of the LLL atelectasis.

On the day immediately post-procedure, the patient presented to a local hospital complaining of pain in the left

\footnotetext{
* Corresponding author: Dr Allen J Blaivas, Assistant Professor of Medicine, Department of Pulmonary, Critical Care, and Sleep Medicine, VA New Jersey Health Care System, 385 Tremont Avenue (561/111), East Orange, New Jersey, USA, 07018.

Tel: +9736761000 Extension 1920 Fax: +973-395-7034 E-mail: allen.blaivas@va.gov
} 
Figure 1. Representative CT scan slice showing an area of atelectasis is noted in the superior segment of the left lower lobe.

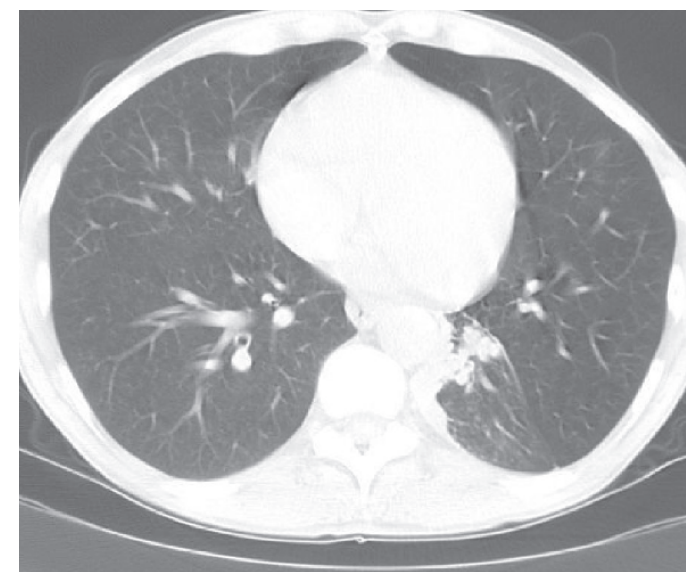

Figure 2. CT scan image displaying resolution of the above noted atelectasis, concomitant with symptom resolution.

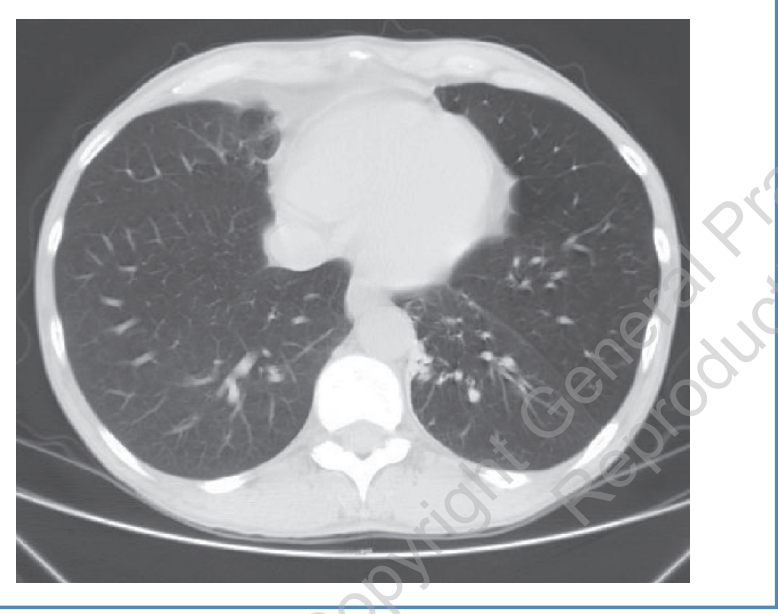

lower chest and back, worse on deep inspiration, with chills, fevers and an increase in yellow sputum production. Chest $x-$ ray showed a new left lower lobe infiltrate. He was treated with moxifloxacin with subsequent improvement.

He was again seen in our pulmonary clinic one month after the bronchoscopy; he had noted a marked increase in dyspnoea and cough over the previous week and was complaining of wheezing. Examination showed him to be in mild respiratory distress with significant diffuse wheezes. He was treated with a course of oral corticosteroids and he improved. A repeat CT scan (Figure 2) was performed after treatment of the COPD exacerbation, which showed that the atelectasis in the LLL had resolved. The patient's clinical symptoms of chest pain, night sweats, and purulent sputum also remitted.

He once again returned to the walk-in clinic in our hospital two months later, again complaining of left lower chest pain,
Figure 3. Recurrence of atelectatic area in the superior segment of the left lower lobe, which occurred with reappearance of the patient's symptoms.

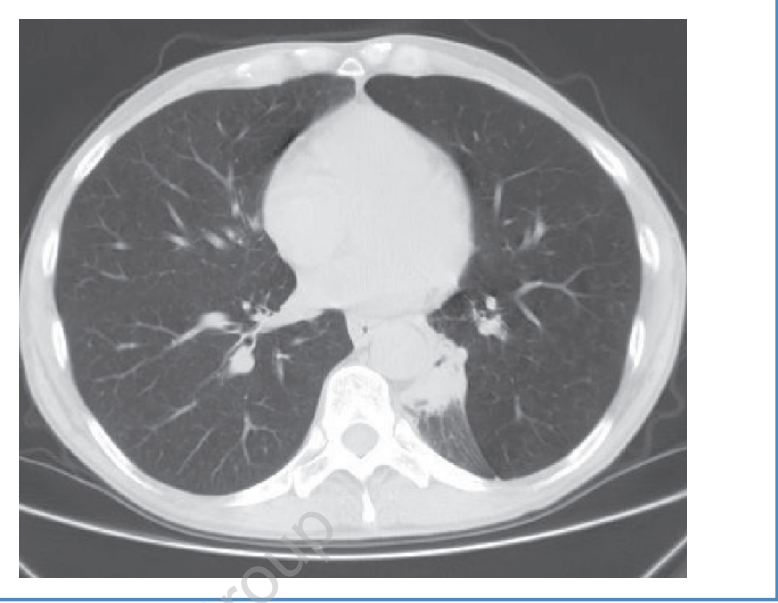

cough, and drenching night sweats. Again he had mild wheezing, particularly in the LLL. Further CT scanning (Figure 3) revealed recurrence of atelectasis in the superior segment of the LLL.

\section{Discussion}

Middle lobe syndrome (MLS) is defined as a recurrent or chronic collapse of the middle lobe of the right lung which typically occurs in children and in asthmatics. Despite the name, it can also occur in other parts of the lung, particularly the lingula and right upper lobe. Sekerel et al reported MLS in all pulmonary segments with the exception of the left upper lobe. ${ }^{1}$

Middle lobe syndrome is generally classified as being obstructive (caused by both extrinsic or intrinsic compression) or non-obstructive. The obstructive type can be caused by diverse aetiologies including endobronchial tumour, granulomatous infections, lymphadenopathy, foreign bodies, endobronchial silicotic nodules, and inspissated mucus associated with cystic fibrosis., ${ }^{2,3}$

The non-obstructive type is typically caused by inflammation, commonly as the result of infection. ${ }^{4,5}$ Benign inflammatory disease has been identified as the most common cause of MLS, accounting for $62 \%$ of cases. ${ }^{5}$ Infectious causes are most commonly bacterial, but can be viral, mycobacterial, or fungal. ${ }^{4,5}$ The most common diagnoses cited as being associated with MLS are bronchiectasis, asthma (particularly in the paediatric population), tuberculosis, ${ }^{6}$ and recurrent pneumonia. Asthma is probably the most well described cause, and MLS is often causative in situations where the asthma is deemed intractable.

We hypothesize that our patient had a "middle lobe-like" syndrome that caused his symptoms, likely on account of his COPD and continued smoking, which led to chronic airway inflammation. Interestingly, upon treatment of his COPD 
exacerbation with oral glucocorticoids his inflammation presumably receded, thus allowing the airway to reopen. Though direct causation is difficult to establish, the fact that LLL atelectasis was present during the COPD exacerbations, and remitted with glucocorticoid treatment, suggests a possible association between COPD and MLS in this patient. In addition, the post-bronchoscopy pneumonia was also likely attributable to lack of drainage of lavage fluid and postprocedure inflammation in this patient's LLL.

Anatomic characteristics, such as the narrow diameter of the lobar bronchus and an acute take-off angle, make the right middle lobe (RML) susceptible to transient, usually partial, obstruction. Such obstruction is considered to be the result of poor drainage of secretions due to inflammation and/or oedema of the RML bronchus. In addition, the relative anatomic isolation of the middle lobe and the poor collateral ventilation decrease the chance of reinflation once atelectasis has been established. These mechanisms help to explain the vicious cycle of recurrent inflammation and obstruction that develops after repeated episodes of infection or asthma exacerbations. ${ }^{7}$ When it occurs in other airway segments it is likely to be due to similar factors.

The most frequent clinical findings are cough, haemoptysis, dyspnoea, chest pain, audible wheezing, fever and chills, most of which were found in our patient. The symptoms are related to the obstructive pneumonia and can be intermittent and recurrent despite antibiotic therapy. ${ }^{4}$

Chest $x$-ray and high resolution CT scan are usually performed to evaluate the involved lung segment. Flexible fibre-optic bronchoscopy is required for diagnosis to evaluate the patency of the involved bronchus, since bronchoscopy may be abnormal in $>40 \%$ of cases. BAL is also recommended to rule out an infectious cause. ${ }^{4}$

Treatment should be directed at the underlying cause. If an obstruction is present, resolution would require removal of the obstruction. In the non-obstructing type, the avoidance of bronchial irritants such as tobacco is likely to be helpful. Bronchodilators and antibiotics may also help resolve the symptoms during an acute event. When secondary to asthma, (and presumably COPD - as in our case), bronchodilators with inhaled or oral corticosteroids may aid resolution. Lobectomy may be necessary and should be performed expeditiously if the patient has persistent unremitting symptoms, with a high likelihood of irreversible disease based on clinical and radiographic findings. ${ }^{2,4,5}$

To our knowledge this is the first case report of COPD causing a middle lobe-like syndrome. A second unusual aspect of this case is the occurrence of post-bronchoscopy pneumonia due to poor drainage in the diseased airway segment. This also has not been previously reported, but should theoretically occur on an occasional basis since one of

\section{Box 1. Key Learning Points:}

- Middle lobe syndrome refers to persistent or recurrent atelectasis of any lung segment caused by either extrinsic or intrinsic airway obstruction, or inflammatory/ infectious etiologies

- The right middle lobe tends to be the most prone to develop collapse due to its relatively narrow diameter and acute take-off angle

- High resolution CT scan and fibre-optic bronchoscopy are usually required to aid diagnosis

- Treatment should be directed at the underlying cause, and medical therapy is usually sufficient. Surgical resection may be beneficial in the appropriately selected patient

the explanations for the propensity of atelectasis of the RML is its poor drainage of secretions.

\section{Conclusion}

MLS is a well known clinical entity that refers to persistent or recurrent atelectasis of any lung segment caused by either extrinsic or intrinsic airway compression, or inflammatory/ infectious aetiologies. HRCT and bronchoscopy are usually required to aid diagnosis. Treatment should be directed at the underlying cause; medical therapy is usually sufficient, but surgery can be beneficial in the appropriate patient. The key learning points from this case are shown in Box 1.

\section{Conflict of interest declaration}

Both Allen Blaivas and Walter Strauss attest that they have no conflicts of interest nor have we received funding relevant to the writing of this manuscript. We also attest to the fact that this manuscript has not been accepted, nor is it under review for publication in another journal.

\section{References}

1. Sekerel BE, Nakipoglu F. Middle lobe syndrome in children with asthma: review of 56 cases. J of Asthma 2004;41:411-17. http://dx.doi.org/10.1081/JAS120033983

2. Ayed AK. Resection of the right middle lobe and lingula in children for middle lobe/lingual syndrome. Chest 2004;125:38-42. http://dx.doi.org/ 10.1378/chest.125.1.38

3. Chien HP, Tao-Ping L, Chen HL, Huang TW. Right middle lobe atelectasis associated with endobronchial silicotic lesions. Arch Path Lab Med 2000; 124:1619-22.

4. Gudmusson G, Gross TJ. Middle lobe syndrome. Am Fam Phys 1996; 53:2547-50.

5. Robinson DA, Lee PC. Middle lobe syndrome: case report and review of literature. Clin Pulm Med 2007;14:244-8.

6. Gupta PP, Gupta KB, Agarwal D. Middle Lobe syndrome due to tuberculous etiology: a series of 12 cases. Ind J of Tuberculosis 2006;53:104-08.

7. Prifitis KN, Mermiri D, Papadopoulou A, et al. The role of timely intervention in the middle lobe syndrome in children. Chest 2005;128:2504-10. http://dx.doi.org/10.1378/chest.128.4.2504 